\title{
Pregravid body mass index is negatively associated with diet quality during pregnancy
}

\author{
Barbara A Laraia ${ }^{1, *}$, Lisa M Bodnar ${ }^{2}$ and Anna Maria Siega-Riz ${ }^{3}$ \\ ${ }^{1}$ Department of Medicine, Center for Health and Community, University of California, San Francisco, CA 941 18, \\ USA: ${ }^{2}$ Department of Epidemiology, Graduate School of Public Health, University of Pittsburgh, Pittsburgh, PA, USA: \\ ${ }^{3}$ Departments of Epidemiology, and Nutrition, School of Public Health, Carolina Population Center, University of \\ North Carolina, Chapel Hill, NC, USA
}

Submitted 20 April 2006: Accepted 20 November 2006: First published online 19 February 2007

\begin{abstract}
Objective: To investigate the association between pregravid weight status and diet quality.

Design: Institute of Medicine body mass index (BMI) cut-off points of $<19.8 \mathrm{~kg} \mathrm{~m}^{-2}$ for underweight, $19.8-26.0 \mathrm{~kg} \mathrm{~m}^{-2}$ for normal weight, $>26.0-29.0 \mathrm{~kg} \mathrm{~m}^{-2}$ for overweight and $>29 \mathrm{~kg} \mathrm{~m}^{-2}$ for obese were used to categorise women's weight status. Dietary information was obtained by self-report at 26-28 weeks' gestation using a modified Block food-frequency questionnaire. The Diet Quality Index for Pregnancy (DQI-P) included: servings of grains, vegetables and fruits, folate, iron and calcium intake, percentage calories from fat, and meal pattern score. Multinomial logistic regression models were used to estimate the association between weight status and tertiles of DQI-P controlling for potential individual confounders.

Setting: A clinical-based population recruited through four prenatal clinics in central North Carolina.

Subjects: A total of 2394 women from the Pregnancy, Infection and Nutrition study were included in this analysis.

Results: Evidence of a dose-response relationship was found between BMI and inadequate servings of grains and vegetables, and iron and folate intake. Pregravid obesity was associated with $76 \%$ increased odds of falling into the lowest diet quality tertile compared with underweight women after controlling for potential confounders.

Conclusion: A modest association was found between pregravid weight status and diet quality. If corroborated, these findings suggest that overweight pregnant women should be targeted for nutrition counselling interventions aimed to improve diet quality.
\end{abstract}

A woman's nutritional status prior to and during pregnancy affects foetal growth and development and the course of her pregnancy, as well as her long-term health status ${ }^{1-6}$. Pre-pregnancy weight status, in particular, has been shown to be a primary indicator of women's nutritional status and a predictor for reproductive health ${ }^{7,8}$. Pregravid overweight and obesity have been associated not only with excessive weight gain?, but also with pregnancy complications such as Caesarean section ${ }^{10}$, gestational diabetes ${ }^{11}$, pre-eclampsia ${ }^{12}$, pregnancyinduced hypertension ${ }^{13}$ and postpartum anaemia ${ }^{14}$. With regards to infant outcomes, pregravid overweight and obesity status has been independently associated with macrosomia ${ }^{10,15}$, neural tube defects ${ }^{16}$ and infant mortality $^{10,17}$. Most of these maternal and infant outcomes are influenced by diet as well, but the impact of diet may be overshadowed by the strong association of current weight status.

While the association of pre-pregnancy overweight with adverse outcomes is clear and striking, the mechanism(s) underlying this association remain obscure. In particular, the importance of diet quality to these outcomes is unknown. Diet could directly lead to adverse pregnancy outcome due to marginal nutrient deficiencies (e.g. low iron intake leading to iron-deficiency anaemia, or low folate intake leading to neural tube defects or preterm birth) ${ }^{2,3}$, through nutrient excess (e.g. high fat and low carbohydrate intake) and gestational diabetes ${ }^{18}$ or through indirect influence on foetal outcomes mediated by maternal weight gain $^{19}$. Alternatively, non-diet factors (e.g. exercise/physical activity) might be as or more important. If rational public health strategies are to be 
developed to improve outcomes for obese pregnant women, it will be critical first to understand the relationship between weight status and diet quality in this population.

On this evidence, we hypothesised that pre-pregancy body mass index (BMI) would be inversely associated with diet quality in pregnancy. In this report, we model the association of pregravid BMI and diet quality among pregnant women in a prospective cohort study, controlling for sociodemographic, health and physical activity behaviours.

\section{Subjects and methods}

\section{Sample and data collection}

This study used data from the Pregnancy, Infection and Nutrition (PIN) cohort, a prospective study of determinants of preterm birth. Participants were predominantly lower- to middle-income women who received prenatal care at the University of North Carolina Residents' and Private Physicians' Obstetrics Clinics, the Wake County Department of Human Services and Wake Area Health Education Center Prenatal Care Clinics. Between 1995 and 2000, 3163 women were recruited into the study at between 24 and 29 weeks' gestation. A self-administered food-frequency questionnaire was completed between 26 and 28 weeks' gestation followed by a telephone interview at 26-31 weeks' gestation that solicited information on sociodemographic characteristics, health behaviours and previous as well as current medical history. A total of 2394 PIN participants with a pregravid BMI value and information on diet were included in this analysis. Women with incomplete dietary and BMI information had a lower mean age (24.9 versus 26.4 years), mean education (12.5 versus 13.6 years) and mean income (188 versus $257 \%$ poverty), and a higher proportion were black compared with women with complete data who were included in the analysis ( $P=0.001$ for each attribute). The procedures followed for this study were in accordance with the ethical standards of the Institutional Review Board of the University of North Carolina School of Medicine and Wake Medical Center.

\section{Definition of outcome}

Dietary intake was assessed using a 120-item modified Block food-frequency questionnaire (FFQ). The modified FFQ captured usual diets reflective of the previous 3month period, corresponding to the second trimester of pregnancy instead of the previous 12-month period; it included foods typical of a southern cuisine; and it excluded information on dietary supplements as a more detailed set of questions were added to the study questionnaire $^{20}$. Based on dietary data generated from the FFQ, a Diet Quality Index for Pregnancy (DQI-P) was constructed $^{17}$. The DQI-P was based on eight dietary components - percentage of recommended servings per day of grains, vegetables and fruits, percentage meeting less than the Estimated Average Requirement (EAR) of folate and iron, percentage meeting less than Adequate Intake (AI) of calcium, percentage of calories from fat, and a meal pattern score. The first three components reflect the dietary adequacy of grain, vegetable and fruit intakes based upon the 2000 Dietary Guidelines for Americans ${ }^{21}$ and the Food Guide Pyramid ${ }^{22}$. The next three components of the DQI-P reflect intake of nutrients particularly important for pregnancy: folate, iron and calcium. These nutrients represent dietary intake exclusive of vitamin/mineral supplements. The seventh component was percentage of energy from fat in the diet, based on recommendations from the Dietary Guidelines for Americans ${ }^{21}$. The final component relates to meal/snack patterning. The Institute of Medicine (IOM) recommends that women should follow a meal pattern of three meals and at least two snacks during gestation ${ }^{5}$. The number of meals and snack was asked as part of the FFQ. Previous research has shown that meal patterns of pregnant women and the frequency of food intake during pregnancy are relevant to the relationship between maternal nutrition status and preterm birth ${ }^{4,23}$. Diet composition measures have been found to be internally valid such that both the foods and nutrients that comprise the index and those that are not directly measured by it increase as the index or score increases ${ }^{20,24}$. A categorical variable was created using DQI-P tertiles as cut-off points to compare the lowest with the highest tertile.

\section{Definition of covariates}

Self-reported pre-pregnancy weight and measured height were used to construct the BMI. Recalled pre-pregnancy weight is shown to correlate well with measured weights ${ }^{25}$. An imputed weight was used in lieu of the self-reported measure only when it was missing or considered biologically implausible. This imputed weight was calculated using the measured weight at the first prenatal visit (if taken prior to 16 weeks) minus the recommended amount of weight to be gained in the first and second trimesters as defined by the $\mathrm{IOM}^{6}$. This methodology has been used previously by our group ${ }^{1,26}$. If the first weight measurement was after 15 weeks of gestation, a pre-pregnancy weight could not be imputed. Weight status categories for this analysis were defined using the IOM BMI cut-off points for pregnant women as follows: $<19.8 \mathrm{~kg} \mathrm{~m}^{-2}$ (underweight), $19.8-$ $26.0 \mathrm{~kg} \mathrm{~m}^{-2}$ (normal), $>26-29.0 \mathrm{~kg} \mathrm{~m}^{-2}$ (overweight) and $>29 \mathrm{~kg} \mathrm{~m}^{-2}$ (obese) ${ }^{5}$. These BMI cut-off points were used because gestational weight gain recommendations are based on these categories. The underweight category was used as the referent category because (1) the majority of women (63.5\%) who were categorised as underweight had BMI values between 18.5 and $19.8 \mathrm{~kg} \mathrm{~m}^{-2}$, which is classified as normal weight by the World Health Organization $^{27}$; (2) the relationship between foods and 
nutrients and weight status appeared to be a doseresponse relationship; and (3) the underweight group had the highest mean diet quality score.

\section{Statistical analysis}

The statistical analysis was based on a total of 2394 women who had complete dietary, weight and height data. Descriptive statistics for the main variables of interest were generated. Analysis of variance with Bonferroni correction and non-parametric test for trend were conducted for each of the eight DQI-P components - percentage of recommended servings per day of grains, vegetables and fruits, percentage meeting less than the EAR of folate and iron, percentage meeting less than the AI of calcium, percentage of calories from fat, and a meal pattern score and DQI-P score across strata of BMI. The crude relative risk and 95\% confidence interval (CI) for the association between pregravid weight status and DQI-P tertile was calculated. Multinomial logistic regression was used to estimate the conditional association of pregravid BMI status with diet quality, controlling for individual characteristics. As previously mentioned, the diet quality variable was categorised into tertiles using the highest tertile as the reference group. Potential confounders previously reported in the literature that might affect a woman's diet quality and overweight status included age, race (non-Hispanic white, non-Hispanic black, other), level of education ( $\leq$ high school, high school, some college, $>$ college), percentage poverty, number of children (no previous children, one child, more than one child), married (yes/no), any smoking during pregnancy (yes/no), regular vitamin use prior to pregnancy (yes/no) and vigorous leisure activity 3 months prior to pregnancy (yes/no). STATA software was used for data management and statistical calculations ${ }^{28}$.

\section{Results}

PIN participants for this analysis were 56\% non-Hispanic white, 36\% non-Hispanic black and 6\% other race; $52 \%$ were married; $29 \%$ had no previous children; and the average age was 26 (range, 15-45) years. Fifty-five per cent had an income of $<185 \%$ of the poverty level (the income eligibility criterion for the Special Supplemental Nutrition Program for Women, Infants, and Children) and $49 \%$ had less than a high school education. Behavioural characteristics indicated that $79 \%$ of PIN participants took a vitamin/mineral supplement regularly during pregnancy, $17 \%$ engaged in vigorous leisure activity prior to pregnancy and 39\% smoked at any time during pregnancy. The percentages of women in each BMI category (underweight, normal, overweight and obesity) were $15,46,12$ and $27 \%$, respectively.

The mean DQI-P was 55 (standard deviation (SD 11.6). By tertile, mean DQI-P scores were: 42 (SD 7.2), for the lowest; 56 (SD 3.0), for the middle; and 68 (SD 4.2) for the highest. Mean DQI-P score varied significantly by sociodemographic characteristics in bivariate analyses (Table 1), consistent with our previously published data ${ }^{20}$. Interestingly, significantly higher mean DQI-P scores were found for women who engaged in vigorous prepregnancy leisure activity and pre-pregnancy vitamin use compared with women who did not engage in vigorous pre-pregnancy leisure activity or who did not use vitamins (Table 1).

Table 2 shows the proportion of women meeting the suggested dietary recommendation during pregnancy by BMI status for each DQI-P component. As BMI status increased, the average percentage of grain and fruit servings, and the proportion of women who met IOMsuggested meal pattern decreased, while the proportion of women who did not meet the EAR for iron and folate increased. The average percentage of grain and vegetable servings, the percentage of energy from fat, EAR for iron and folate, and meal patterning varied by BMI. Obese women had significantly lower intakes of vegetable

Table 1 Mean DQI-P scores and SD by health behaviour characteristics

\begin{tabular}{|c|c|c|}
\hline Maternal characteristics & $n$ & $\begin{array}{l}\text { Mean DQI-P } \\
\text { (SD) }\end{array}$ \\
\hline \multicolumn{3}{|l|}{ Ethnicity } \\
\hline White (reference) & 1402 & $54.3(11.1)$ \\
\hline Black & 977 & $55.5(12.4)^{\star}$ \\
\hline Other & 161 & $58.3(11.1)^{*}$ \\
\hline \multicolumn{3}{|l|}{ Income } \\
\hline$\leq 185 \%$ poverty (reference) & 1336 & $54.6(12.3)$ \\
\hline$>186 \%$ poverty & 1043 & $55.8(10.6)^{\star}$ \\
\hline \multicolumn{3}{|l|}{ Marital status } \\
\hline $\begin{array}{l}\text { Separated, divorced, widowed } \\
\text { (reference) }\end{array}$ & 214 & $53.3(12.5)$ \\
\hline Single & 1019 & $54.6(12.2)$ \\
\hline Married & 1307 & $55.6(11.0)^{*}$ \\
\hline \multicolumn{3}{|l|}{ Education } \\
\hline$<$ High school (reference) & 467 & $52.2(12.5)$ \\
\hline High school & 778 & $54.4(12.0)^{\star}$ \\
\hline College & 604 & $55.4(11.3)^{\star}$ \\
\hline$>$ College & 690 & $57.3(10.4)^{\star}$ \\
\hline \multicolumn{3}{|l|}{ Children } \\
\hline More than one (reference) & 1109 & $54.1(11.7)$ \\
\hline One child & 684 & $55.4(11.8)$ \\
\hline No previous children & 739 & $56.0(11.4)^{*}$ \\
\hline \multicolumn{3}{|l|}{ Age (years) } \\
\hline$\leq 20$ (reference) & 451 & $53.8(12.4)$ \\
\hline$>20-25$ & 721 & $54.5(11.7)$ \\
\hline$>25-30$ & 645 & $54.9(11.7)$ \\
\hline$>30$ & 723 & $56.3(11.0)^{*}$ \\
\hline \multicolumn{3}{|l|}{ Smoking } \\
\hline Yes (reference) & 1004 & $53.5(11.7)$ \\
\hline No & 1535 & $56.0(11.7)^{\star}$ \\
\hline \multicolumn{3}{|l|}{ Physical activity } \\
\hline $\begin{array}{l}\text { No pre-pregnancy vigorous } \\
\text { leisure activity }\end{array}$ & 2093 & $54.3(11.8)$ \\
\hline $\begin{array}{l}\text { Pre-pregnancy vigorous } \\
\text { leisure activity }\end{array}$ & 447 & $58.3(10.1)^{*}$ \\
\hline \multicolumn{3}{|l|}{ Vitamin use } \\
\hline None before pregnancy & 1941 & $54.3(11.8)$ \\
\hline Any before pregnancy & 534 & $57.4(10.4)^{\star}$ \\
\hline
\end{tabular}

DQI-P - Diet Quality Index for Pregnancy; SD - standard deviation.

* Significant at $P<0.001$, using analysis of variance with Bonferroni correction. 
servings, and lower iron and folate intakes than underweight women, and higher intakes of percentage energy from fat than normal weight women. Obese women had a significantly lower overall DQI-P score than both underweight and normal weight women.

Multinomial logistical regression analysis results show that women classified as obese had increased odds of falling into the lowest versus the highest diet quality tertile compared with underweight women (odds ratio $(\mathrm{OR})=1.87(95 \%$ CI 1.37-2.55)) (Table 3). We then compared two models controlling first for variables hypothesised as exogenous to the BMI and diet quality equation by virtue of the fact that diet quality could not influence their status (e.g. diet quality does not influence age or race). In the second model, we controlled for the exogenous variables as well as pre-pregnancy vigorous leisure activity and vitamin use. These variables could be influenced by diet quality and, furthermore, prepregnancy vigorous leisure activity determines pregravid BMI. Nonetheless, we controlled for these variables in a final model because they may be indicators of health behaviour patterns that would be predictive of diet quality during pregnancy. The inverse association between pregravid obesity and poor diet quality remained significant when we adjusted for socio-economic and smoking variables compared with underweight women (adjusted OR $=1.86$ (95\% CI 1.32-2.62)) and persisted even after we controlled for pre-pregnancy vitamin use and pre-pregnancy vigorous leisure activity (adjusted $\mathrm{OR}=1.76$ (95\% CI 1.24-2.49))

Finally, we estimated models using normal weight as the referent category. Women of underweight status had evidence of a 23\% decreased odds of being in the poorest diet quality tertile (OR $=0.77$ (95\% CI 0.58-1.02)), while no difference was found for overweight women, and obese women were at $44 \%$ increased odds of being in the poorest diet quality tertile $(\mathrm{OR}=1.44$ (95\% CI 1.13-1.84)) compared with women of normal weight. This pattern persisted for both adjusted models; underweight women were at 28\% decreased odds (adjusted OR $=0.72$ (95\% CI $0.53-0.99)$ ) and obese women were at $27 \%$ increased odds (adjusted OR = 1.27 (95\% CI 0.97-1.66)), although slightly attenuated.

\section{Discussion}

The study of dietary patterns is complex, and the creation of dietary indices is one way of overcoming this problem. For example, although the association between folate and neural tube defects has been well established, there are additional nutrients such as zinc, vitamin $\mathrm{B}_{6}$ and methionine that may influence neural tube defect risk. Therefore, understanding the association of these nutrients, measured by their composite score, may be important. Carmichael et al. have shown an association between neural tube defects and a dietary quality score 
Table 3 Crude and adjusted odds ratios for being in the lowest compared with the highest DQI-P tertile by BMI status

\begin{tabular}{|c|c|c|c|c|}
\hline Characteristics & Level & Model $1^{*}$ & Model 2† & Model 3‡ \\
\hline \multirow[t]{4}{*}{ Weight status } & Obese & $1.87(1.37-2.55)$ & $1.86(1.32-2.62)$ & $1.76(1.24-2.49)$ \\
\hline & Overweight & $1.29(0.88-1.88)$ & $1.32(0.88-1.99)$ & $1.31(0.87-1.99)$ \\
\hline & Normal weight & $1.29(0.98-1.72)$ & $1.43(1.05-1.94)$ & $1.38(1.01-1.89)$ \\
\hline & Underweight & 1.00 & 1.00 & 1.00 \\
\hline \multirow[t]{4}{*}{ Education level } & $<$ High school & & $2.40(1.53-3.76)$ & $2.15(1.35-3.40)$ \\
\hline & High school & & $1.94(1.34-2.80)$ & $1.84(1.26-2.68)$ \\
\hline & Some college & & $1.39(0.98-2.00)$ & $1.38(0.96-1.99)$ \\
\hline & College & & 1.00 & 1.00 \\
\hline \multirow[t]{3}{*}{ Race } & Other & & $0.45(0.28-0.72)$ & $0.45(0.28-0.72)$ \\
\hline & Black & & $0.59(0.45-0.77)$ & $0.57(0.44-0.76)$ \\
\hline & White & & 1.00 & 1.00 \\
\hline Maternal age (years) & & & $0.98(0.96-1.00)$ & $0.98(0.96-1.01)$ \\
\hline \multirow{3}{*}{ Children } & $>1$ child & & $1.56(1.17-2.09)$ & $1.50(1.12-2.02)$ \\
\hline & 1 child & & $1.11(0.83-1.48)$ & $1.10(0.82-1.48)$ \\
\hline & No children & & 1.00 & 1.00 \\
\hline Married & Yes versus no & & $1.06(0.81-1.38)$ & $1.02(0.78-1.34)$ \\
\hline Any smoking & Yes versus no & & $1.29(1.02-1.62)$ & $1.28(1.01-1.63)$ \\
\hline Pre-pregnancy vitamin use & Yes versus no & & & $0.68(0.51-0.89)$ \\
\hline Pre-pregnancy vigorous leisure activity & Yes versus no & & & $0.67(0.50-0.90)$ \\
\hline
\end{tabular}

DQI-P - Diet Quality Index for Pregnancy; BMI - body mass index.

${ }^{*}$ Crude association between BMI status and DQI-P tertiles.

† Adjusted for exogenous variables.

$\ddagger$ Adjusted for exogenous variables, pre-pregnancy vigorous leisure activity and vitamin use.

that was based on intakes of calcium, folate, iron, vitamin $\mathrm{B}_{6}$ and vitamin $\mathrm{A}$, and percentage of calories from fat and from sweets ${ }^{3}$. Although public health importance is being placed on capturing diet quality, there are few appropriate instruments to measure diet quality well during pregnancy. Recently, the Healthy Eating Index (HEI), a foodbased index, was tested for its reliability to measure these important nutrients during pregnancy but was not found to be sufficient ${ }^{29}$. For example, macronutrient intake calculated from the food-based HEI was similar for pregnant and non-pregnant women; however, micronutrient intakes among pregnant women for iron and folate were exceedingly low, with only $4 \%$ of pregnant women meeting the folate recommendation and none meeting the iron recommendation. This might be due to a dependence on supplements during pregnancy, which are not part of the HEI, to meet recommendations, or due to the inadequacy of the food-based HEI to pick up micronutrient intake for pregnant women ${ }^{29}$.

This study showed that the DQI-P and several of its components decreased with increasing BMI. Pre-pregnancy weight status may have profound influences on diet during pregnancy as it is a predictor of gestational weight gain which is associated with preterm birth ${ }^{30}$. In a recent study of Belgium women, differences were found in dietary behaviour between pregnant and non-pregnant women. Pregnant women reported higher intakes of fruits, fibre, beef, dairy and fat, and reduced intake of raw vegetables for food safety reasons. Although the findings do not differentiate by weight status, the authors concluded that pregnant women may make conscious efforts to improve their diet and that pregnancy can be viewed as an opportunity for positive dietary change ${ }^{31}$. In our study, women who were obese had a significantly lower diet quality than all other women. An assessment of the eight food and nutrient components of the DQI-P showed that the percentage of grain and fruit servings, the proportion of women who met the IOM meal pattern recommendation, and the proportion of women meeting the EAR for iron and folate all decreased as BMI increased. A modest relationship between pregravid obesity and poor diet quality remained after adjusting for several traditional socio-economic variables and smoking when compared with underweight women. After adjusting for pre-pregnancy vigorous leisure activity and vitamin use, the association remained significant. Physical activity is an important determinant of BMI and part of the energy balance equation. Controlling for pre-pregnancy vigorous leisure activity and vitamin use may overspecify the model and introduce bias. However, since these indicators might capture health behaviours that may explain a woman's diet quality during pregnancy, we adjusted for these to estimate the association of pregravid BMI and diet quality in the final models.

Overweight has been associated with iron deficiency anaemia among women of reproductive age in the general population $^{32}$. Pregnancy overweight and obesity have been found to be associated with postpartum anaemia ${ }^{14}$. Our findings, that as BMI increased so did the proportion of women who did not meet the EAR for iron, suggest that dietary iron might contribute to low iron stores for overweight women during and after pregnancy.

Our finding that dietary folate intake differs by BMI status is concerning due to the increased risk of several birth defects associated with being obese ${ }^{33}$. However, Shaw et al. did not find lower intakes of dietary folate 
among obese women in their study that assessed neural tube defect risk in a Californian population ${ }^{34}$. One recent British study found that pre-pregnancy BMI was inversely associated with nutrient intakes of folate as well as fibre ${ }^{35}$. More recently, low diet quality was found to be associated with increased risk of neural tube defects among women who were not obese in a case-control study in a Californian clinic population ${ }^{3}$.

The modest association between BMI and diet quality might represent a true relationship between weight status and diet quality, or the relationship might be masked due to under-reporting of grain servings from the FFQ. We have previously commented on the low reporting of grain servings for this population of pregnant women; however, there are no studies that report adequacy of pregnant women meeting the Food Guide Pyramid recommendations for grains ${ }^{20}$.

The results presented here of a modest association between BMI and diet quality warrant further study of diet adequacy among pregnant women in light of the growing obesity epidemic. If such an association is confirmed in future studies, obese pregnant women should be targeted for nutrition counselling and interventions aimed at improving overall diet quality, with particular emphasis on frequency of meals and snacks, carbohydrate and micronutrient intake.

\section{Acknowledgements}

Sources of funding: This study was supported by grants HD28684 and HD28684A from the National Institute of Child Health and Human Development, National Institutes of Health; funding from the National Institutes of Health, General Clinical Research Centers program of the Division of Research Resources (grant \# RR00046).

Authorship responsibilities: B.A.L. conceptualised the research question for this study, conducted the statistical analysis and interpretation of the results, and prepared the manuscript for publication. L.M.B. created the DQI-P index, assisted with the conceptualisation of the research question, interpretation of results and writing of the manuscript. A.M.S.-R. is a co-investigator of the PIN study. She contributed to the study design, implementation and data collection of the PIN study and aided in the conceptualisation of the research question, statistical analysis and writing of the manuscript.

Acknowledgements: We greatly appreciated the work of and assistance given to us by Diane Kaczor. We are grateful for the cooperation and support of all study staff members, prenatal care providers and particularly the women who participated in this study.

Conflict of interest declaration: None of the authors has any conflict of interest regarding the study, analysis or results of this manuscript.

\section{References}

1 Siega-Riz AM, Adair LS, Hobel CJ. Maternal underweight status and inadequate rate of weight gain in the third trimester of pregnancy increases the risk of preterm delivery. Journal of Nutrition 1996; 126: 146-53.

2 Siega-Riz AM, Savitz DA, Zeisel SH, Thorp JM, Herring A. Second trimester folate status and preterm birth. American Journal of Obstetrics and Gynecology 2004; 191: 1851-7.

3 Carmichael SL, Shaw GM, Selvin S, Schaffer DM. Diet quality and risk of neural tube defects. Medical Hypotheses 2003; 60: $351-5$.

4 Siega-Riz AM, Herrmann TS, Savitz DA, Thorp J. The frequency of eating during pregnancy and its effect on preterm delivery. American Journal of Epidemiology 2001; 153: $647-52$

5 Institute of Medicine. Nutrition During Pregnancy and Lactation: An Implementation Guide. Committee on Nutritional Status during Pregnancy and Lactation. Food and Nutrition Board, Subcommittee for Clinical Application Guide. Washington, DC: National Academy Press, 1992.

6 Institute of Medicine. Nutrition During Pregnancy. Part I, Weight Gain. Washington, DC: National Academy Press, 1990.

7 Siega-Riz AM, Promislow JE, Savitz DA, Thorp JM, McDonald T. Vitamin $\mathrm{C}$ intake and the risk of preterm delivery. American Journal of Obstetrics and Gynecology 2003; 189: $1-7$.

8 Philipps C, Johnson NE. The impact of quality of diet and other factors on birth weight of infants. American Journal of Clinical Nutrition 1977; 30: 215-25.

9 Lederman SA, Alfasi G, Deckelbaum RJ. Pregnancyassociated obesity in black women in New York City. Maternal and Child Health Journal 2002; 6: 37-42.

10 Vahratian A, Siega-Riz AM, Savitz DA, Zhang J. Maternal prepregnancy overweight and obesity and the risk of cesarean delivery in nulliparous women. Annals of Epidemiology 2005; 15: 467-74.

11 Solomon CG, Willett WC, Rich-Edwards J, Hunter DJ, Colditz GA, Stampfer MJ, et al. A prospective study of pregravid determinants of gestational diabetes mellitus. Journal of the American Medical Association 1997; 278: 1078-83.

12 Bodnar LM, Ness RB, Markovic N, Roberts JM. The risk of preeclampsia rises with increasing prepregnancy body mass index. Annals of Epidemiology 2005; 15: 475-82.

13 Thadhani R, Stampfer MJ, Hunter DJ, Manson JE, Solomon CG, Curhan GC. High body mass index and hypercholesterolemia: risk of hypertensive disorders of pregnancy. Obstetrics and Gynecology 1999; 94: 543-50.

14 Bodnar LM, Siega-Riz AM, Cogswell ME. High prepregnancy BMI increases the risk of postpartum anemia. Obesity Research 2004; 12: 941-8.

15 Ehrenberg HM, Mercer BM, Catalano PM. The influence of obesity and diabetes on the prevalence of macrosomia. American Journal of Obstetrics and Gynecology 2004; 191: 964-8.

16 Werler MM, Louik C, Shapiro S, Mitchell AA. Prepregnant weight in relation to risk of neural tube defects. Journal of the American Medical Association 1996; 275: 1089-92.

17 Cnattingius S, Bergstroumlum R, Lipworth L, Kramer MS. Prepregnancy weight and the risk of adverse pregnancy outcomes. New England Journal of Medicine 1998; 338: $147-52$.

18 Saldana TM, Siega-Riz AM, Adair LS. Effects of macronutrient intake on the development of glucose intolerance during pregnancy. American Journal of Clinical Nutrition 2004; 70: 479-86.

19 Shaw GM, Todoroff K, Carmichael SL, Schaffer DM, Selvin S. Lowered weight gain during pregnancy and risk of neural 
tube defects among offspring. International Journal of Epidemiology 2001; 30: 60-5.

20 Bodnar LM, Siega-Riz AM. A diet quality index for pregnancy captures variation in diet and differences in sociodemographic characteristics. Public Health Nutrition 2002; 5: 801-9.

21 US Department of Agriculture. Dietary Guidelines for Americans, 5th ed. [online], 2000. Available at http://www. usda.gov/cnpp/DietGd.pdf. Accessed 29 January 2001.

22 Shaw A, Fulton L, Savis C, Hogbin M. Using the Food Guide Pyramid: a resource for nutrition educators [online], 1992. Available at http://www.nal.usda.gov/fnic/Fpyr/guide.pdf. Accessed 29 January 2001

23 Herrmann TS, Siega-Riz AM, Hobel CJ, Aurora C, DunkelSchetter C. Prolonged periods without food intake during pregnancy increase risk for elevated maternal corticotropinreleasing hormone concentrations. American Journal of Obstetrics and Gynecology 2001; 185: 403-12.

24 Kant AK, Schatzkin A, Graubard BI, Schairer C. A prospective study of diet quality and mortality in women. Journal of the American Medical Association 2000; 283: 2109-15.

25 Stevens-Simon C, Roghmann K, Mcanarney E. Relationship of self-reported prepregnant weight and weight gain during pregnancy to maternal body habitus and age. Journal of the American Dietetic Association 1992; 92 $85-7$.

26 Siega-Riz AM, Adair LS, Hobel CJ. Institute of Medicine maternal weight gain recommendations and pregnancy outcome in a predominantly Hispanic population. Obstetrics and Gynecology 1994; 84: 565-73.
27 World Health Organization (WHO). Obesity: Preventing and Managing the Global Epidemic: Report of a WHO Consultation on Obesity, Geneva, 3-5 June 1997. Geneva: WHO, 1998.

28 StataCorp. Stata Statistical Software: Release 8.0. College Station, TX: Stata Corporation, 2003.

29 Pick ME, Edwards M, Moreau D, Edmond EA. Assessment of diet quality in pregnant women using the Healthy Eating Index. Journal of the American Dietetic Association 2005; 105: 240-6.

30 Dietz PM, Callaghan WM, Cogswell ME, Morrow B, Ferre C, Schieve LA. Combined effects of prepregnancy body mass index and weight gain during pregnancy on risk of preterm delivery. Epidemiology 2006; 17: 170-7.

31 Verbeke W, Bourdeaudhuij ID. Dietary behavior of pregnant versus non-pregnant women. Appetite 2007; 48: 78-86.

32 Ramakrishnan U, Kuklina E, Stein A. Iron stores and cardiovascular disease risk factors in women of reproductive age in the United States. American Journal of Clinical Nutrition 2002; 76: 1256-60.

33 Watkins ML, Rasumssen SA, Honein MA, Botto LD, Moore CA. Maternal obesity and risk for birth defects. Pediatrics 2003; 111: S1152-7.

34 Shaw GM, Velie EM, Schaffer D. Risk of neural tube defectaffected pregnancies among obese women. Journal of the American Medical Association 1996; 275: 1127-8.

35 Derbyshire E, Davies J, Costarelli V, Dettmar P. Prepregnancy body mass index and dietary intake in the first trimester of pregnancy. Journal of Human Nutrition and Dietetics 2006; 19: $267-73$. 